\title{
Um estudo sobre a distribuição das transferências para o setor de saúde no Brasil
}

\author{
Juliana Barby Simão \\ Mestre em Economia - Fundação Getúlio Vargas (EESP-FGV/SP) \\ Endereço: Rua Itapeva, 474 - São Paulo/SP - Brasil \\ CEP: 01332-000 - E-mail: julianab@gmail.com \\ Veronica Ines Fernandez Orellano \\ Professora - Escola de Economia de São Paulo da Fundação Getúlio Vargas (EESP-FGV/SP) \\ Endereço: Rua Itapeva, 474 - Sala 1005 - São Paulo/SP - Brasil \\ CEP: 01332-000 - E-mail: veronica.orellano@fgv.br \\ Recebido em 08 de janeiro de 2014. Aceito em 31 de julho de 2014.
}

\section{Resumo}

Este trabalho investiga a existência de uma relação positiva entre receita municipal per capita - que inclui arrecadação local de impostos e transferências intergovernamentais incondicionais - e o recebimento de recursos através do Sistema Único de Saúde (SUS) pelos municípios brasileiros. Considerando o aspecto redistributivo, essa relação não seria desejável, já que municípios com maior capacidade de financiamento receberiam mais repasses por habitante destinados à saúde. Foram estimados modelos de painel com efeitos fixos, utilizando dados dos municípios brasileiros no período 2002-2010, obtidos em sua maioria através do Departamento de Informações do SUS (DATASUS) e do Sistema de Informações sobre Orçamentos Públicos em Saúde (SIOPS). Os resultados apontam que a receita municipal exerce, de fato, um impacto positivo inesperado sobre o recebimento de transferências do SUS pela modalidade fundo a fundo. Também foram identificados indícios de que pode haver fatores políticos influenciando o recebimento de recursos do SUS.

\section{Palavras-Chave}

Transferências intergovernamentais. Saúde. Distribuição. Flypaper effect.

\begin{abstract}
This paper investigates the existence of a positive relationship between municipal per capita revenues - which includes local taxes and unconditional lump sum federal grant receipts - and federal grants received through the Unified Health System (Sistema Único de Saúde - SUS) by the brazilian municipalities. Considering the redistributive aspect, this relationship may not be socially desirable, since richer municipalities would benefit from greater grant receipts destined to health care. Fixed effect panel data models were estimated, using municipal information from 2002 to 2010. Data was mostly obtained from Departamento de Informações do SUS (DATASUS) and Sistema de Informações sobre Orçamentos Públicos em Saúde (SIOPS), both from the Brazilian Ministry of Health. Our results show that municipal revenue has indeed a positive and unexpected impact on federal grant receipts destined to health care. We also identified some evidence that there may be political factors influencing the federal grants received through the SUS.
\end{abstract}




\section{Keywords}

Federal grant receipts. Health care. Distribution. Flypaper effect.

\section{JEL Classification}

$\mathrm{HO}, \mathrm{H} 51, \mathrm{H} 77$.

\section{Introdução}

A Constituição Federal de 1988 estabeleceu as bases do atual federalismo brasileiro e, em particular, fortaleceu os governos municipais. Entretanto, o Brasil é um país de dimensões continentais e grandes disparidades regionais, tanto no que se refere à distribuição da população, como nos aspectos sociais e econômicos, ocorrendo em determinadas regióes um descasamento entre a demanda por bens e serviços públicos e a capacidade local de financiamento. Nesse cenário, o país vem se utilizando fortemente das transferências intergovernamentais. Idealmente, essa seria uma forma de atribuir maior autonomia e responsabilidade aos governos locais, além de redistribuir a capacidade fiscal e a qualidade e quantidade dos serviços públicos e da renda ao longo do território.

Em particular, as transferências intergovernamentais desempenham um papel de destaque na área de saúde no Brasil. Desde a criação do Sistema Único de Saúde (SUS), concebido a partir da Constituição de 1988, a descentralização fiscal e administrativa, além da redistribuição dos recursos entre as regiões brasileiras, são aspectos que vêm sendo perseguidos. Visando a garantir universalidade, equidade e integralidade na assistência à saúde, e sob a ótica de que os governos locais têm mais condições de identificar necessidades específicas, o SUS tornou os municípios os principais responsáveis pela gestão da saúde de sua população. No que se refere ao financiamento, a legislação exige que recursos das três esferas de governo sejam necessariamente aplicados na área da saúde. Assim, para que sejam geridos pelos governos municipais e para que haja uma redistribuição ao longo do território, os recursos são destinados a fundos e transferidos aos estados e municípios.

A maior parte das transferências realizadas através do SUS estão inseridas nas modalidades conhecidas como "fundo a fundo" e são obrigatórias e condicionais. Isso significa que elas são realizadas de forma automática e compulsória e devem, necessariamente, ser apli-

Estud. Econ., São Paulo, vol.45, n.1, p. 33-63, jan.-mar. 2015 
cadas em fins específicos na área da saúde. Além disso, não exigem uma contrapartida financeira dos governos locais. No entanto, este estudo apresenta evidências de que o mecanismo de transferências pode estar induzindo a uma espécie de "exigência de contrapartida" e a eventuais distorções em seu papel redistributivo, embora não explicitamente. Em análises preliminares, identificou-se uma relação positiva, em base per capita, entre transferências fundo a fundo recebidas pelos municípios para a saúde e gastos de recursos próprios em saúde, assim como entre as transferências fundo a fundo e o total de recursos sem fins específicos disponíveis ao município, que inclui a arrecadação local de impostos e as transferências incondicionais recebidas (montante ao qual nos referimos neste artigo como receita municipal). Em princípio, essa relação não seria desejável, pois levaria a uma situação em que os municípios com melhores condições de financiamento poderiam estar recebendo mais recursos para a saúde.

Nesse contexto, o objetivo principal deste trabalho é avaliar empiricamente a relação positiva entre a receita municipal e o recebimento de transferências fundo a fundo do SUS pelos municípios brasileiros. O trabalho discute alguns aspectos redistributivos das transferências do SUS, como também sua sensibilidade às necessidades de saúde dos municípios e a fatores políticos. Desenvolvemos um modelo empírico para explicar alguns determinantes das transferências na área da saúde, utilizando um painel de municípios brasileiros no período de 2002 a 2010. Os dados foram obtidos principalmente através do Departamento de Informações do SUS (DATASUS) e do Sistema de Informações sobre Orçamentos Públicos em Saúde (SIOPS), ambos do Ministério da Saúde.

Este trabalho está organizado em seis seções, além desta introdução. $\mathrm{Na}$ segunda seção trazemos um pequeno histórico do SUS, além de um resumo sobre as regras para financiamento e transferências na área da saúde. Julgamos importante entender o contexto em que o sistema foi criado, o qual demonstra a importância do aspecto redistributivo das transferências. Além disso, para melhor interpretação dos resultados do modelo empírico, é importante conhecer os critérios explícitos que definem essas transferências. Na seção 3 discutimos alguns aspectos teóricos associados às hipóteses que testamos no trabalho e, na seção 4, apresentamos a proposta de análise empírica, bem como os dados utilizados. A seção 5 discute os resultados e, por fim, a seção 6 traz as conclusões e considerações finais. 


\section{Estrutura da assistência e do financiamento público à saúde no Brasil}

A assistência e o financiamento público à saúde no Brasil são realizados por intermédio do Sistema Único de Saúde, o SUS. Como descrevem Souza (Souza 2002) e Mendes, Miranda e Cossio (Mendes, Miranda e Cossio 2008), o sistema, nos moldes em que conhecemos hoje, é resultado de mais de duas décadas de avanços na área de saúde pública brasileira. Ainda assim, o próprio trabalho de Mendes, Miranda e Cossio destaca pontos em que há espaço para discussões e evoluções.

O SUS foi concebido com a promulgação da Constituição Federal de 1988 e institucionalizou a universalidade da assistência pública à saúde no Brasil, procurando trazer um carácter mais redistributivo aos gastos públicos em saúde. A descentralização da gestão é um dos princípios-chave para o funcionamento do sistema, sendo que as políticas e diretrizes são definidas por comissões de que participam as três esferas de governo - federal, estadual e municipal -, mas os recursos do governo federal são transferidos para que as ações sejam executadas pelos estados e, principalmente, pelos municípios.

Antes da implantação do SUS, a cobertura do sistema público federal de saúde estava restrita principalmente aos trabalhadores do mercado formal e a seus dependentes. A assistência médica e hospitalar pública federal era prestada através do INAMPS - Instituto Nacional de Assistência Médica da Previdência Social, uma autarquia do Ministério da Previdência e Assistência Social. Ao Ministério da Saúde cabiam ações como as campanhas de vacinação e de controle de endemias, além da gestão de alguns hospitais especializados nas áreas de psiquiatria e tuberculose, mas não a gestão completa da assistência à saúde da população. Além de não ter um carácter universal, o INAMPS ajudava a acentuar as desigualdades regionais no Brasil. Como a assistência era destinada apenas aos trabalhadores da economia formal, os estados e municípios mais ricos e desenvolvidos, que possuíam maior presença das relações formais de trabalho, eram os que contavam com o maior número de postos de assistência médica e hospitalar. Em dados da Secretaria de Planejamento do INAMPS de 1986, observa-se que, naquela época, gastava-se 59,28\% dos recursos em saúde do país com a região Sudeste, enquanto esta representava somente $43,79 \%$ da população do país. Por outro lado,

Estud. Econ., São Paulo, vol.45, n.1, p. 33-63, jan.-mar. 2015 
eram gastos apenas $18,10 \%$ dos recursos com o Nordeste, que totalizava 28,82\% da população brasileira à época.

Em 1990, com a transferência do INAMPS do Ministério da Previdência para o Ministério da Saúde, a rede de assistência médico-hospitalar financiada pelo governo federal deixou de ser restrita a apenas uma parcela da população brasileira, o que começou a proporcionar a universalidade no atendimento assegurada pela Constituição Federal. Entretanto, a redistribuição dos recursos ao longo das regiões do Brasil ainda levou algum tempo para se concretizar, uma vez que a assistência permaneceu vinculada às instalações do INAMPS. Nesse sentido, foi preciso implementar um sistema de transferências de recursos e responsabilidades entre os entes da federação.

Apenas no ano 2000, com a promulgação da Emenda Constitucional n. ${ }^{\circ} 29$, definiu-se efetivamente qual parcela das receitas da União, dos estados e dos municípios deveria compor os fundos de assistência à saúde. Além disso, as Normas Operacionais do SUS, publicadas nos anos 90 e início dos anos 2000, foram atribuindo maior responsabilidade aos estados e municípios na definição de estratégias e na gestão de recursos. O trabalho de Souza (Souza, 2002) traz uma resenha completa sobre o processo de institucionalização e operacionalização do SUS, bem como um histórico das leis e normativas que constituíram a base do sistema hoje em vigor.

\subsection{Regras de financiamento e transferências}

De acordo com a Emenda Constitucional no 29 (EC-29), ficou estabelecido que a União deveria aplicar, em 2000, o montante empenhado em 1999 acrescido de, no mínimo, 5\%. Para os demais anos, a EC-29 estabelece que a União deve dedicar à saúde um volume de receitas igual ao do ano anterior, corrigido pela variação nominal do Produto Interno Bruto (PIB). Já para os estados e municípios, estipula que devam dedicar, respectivamente, um mínimo de 12\% e 15\% de suas receitas anuais para o financiamento à saúde.

Para efeito de cumprimento da EC-29, a receita dos estados é proveniente de quatro fontes: (i) impostos estaduais (ICMS - sobre a circulação de mercadorias e serviços; IPVA - sobre a propriedade de veículos automotores; ITCMD - sobre heranças e doações), 
(ii) transferências da União (cota-parte do Fundo de Participação dos Estados (FPE); cota-parte do IPI-Exportação; transferências da Lei Complementar $n^{\circ} 87 / 96$ - Lei Kandir), (iii) imposto de renda retido na fonte e (iv) outras receitas correntes, como a receita da dívida ativa de impostos e multas. Para o cálculo da base de receitas dos estados sobre a qual se aplica o mínimo de $12 \%$ para o financiamento à saúde, deve-se subtrair, da soma das fontes listadas acima, o montante em transferências constitucionais e legais que é encaminhado dos estados aos municípios. Já no caso dos municípios, a base para o cálculo dos $15 \%$ sobre a receita é composta pela soma de cinco fontes: (i) impostos municipais (ISS - sobre serviços de qualquer natureza; IPTU - sobre a propriedade predial e territorial urbana; ITBI - sobre a transmissão de bens imóveis), (ii) transferências da União (cota-parte do Fundo de Participação dos Municípios (FPM); cota-parte do ITR; transferências da Lei Complementar no 87/96 Lei Kandir), (iii) imposto de renda retido na fonte, (iv) transferências do Estado (cota-parte do ICMS; cota parte do IPVA; cota-parte do IPI-Exportação) e (v) outras receitas correntes (receita da dívida ativa de impostos, multas, juros e correção monetária de impostos).

Para efeito de fiscalização e controle, a EC-29 estabelece que os estados e municípios devem constituir fundos de saúde para receber os recursos locais e os recursos transferidos a serem aplicados na área de saúde. Além disso, determina a constituição dos Conselhos Estaduais e Municipais de Saúde, que são os responsáveis por fiscalizar a aplicação dos recursos. Desde 2006, quando foi estabelecido o "Pacto pela Saúde", as transferências condicionais especificadas pela EC-29 são destinadas pela União aos governos locais por meio de um dos seguintes blocos de financiamento: (i) Atenção Básica, (ii) Atenção de Média e Alta Complexidade, (iii) Vigilância em Saúde, (iv) Assistência Farmacêutica, (v) Gestão do SUS e (vi) Investimentos. Tais transferências são conhecidas como "fundo a fundo" e ocorrem de forma automática ao longo do ano. Antes do Pacto havia mais de 100 formas de repasses de recursos financeiros, o que trazia algumas dificuldades para sua aplicação (Brasil, 2006). Embora as categorias tenham sido reformuladas, as regras para transferências e financiamento continuaram com características gerais similares ao que já era praticado antes (Mendes, Miranda e Cossio 2008). 
Para o fundo de Atenção Básica são transferidos os recursos do Piso de Atenção Básica Fixo Ampliado, além do Piso de Atenção Básica Variável. O primeiro constitui-se de um valor fixo per capita e deve ser destinado a ações de assistência básica da população, tais como: controles de tuberculose, da hipertensão arterial e do diabetes; eliminação da hanseníase; e programas de saúde bucal, da criança e da mulher. O segundo piso é destinado ao desenvolvimento de programas específicos, tais como o de combate a carências nutricionais, o de Agentes Comunitários de Saúde (PACS) e o de Saúde da Família (PSF). Seu valor depende do nível total de produção ou de cobertura de cada programa, havendo, entretanto, um limite máximo por estado e/ou município. Mendes, Miranda e Cossio (Mendes, Miranda e Cossio, 2008) explicam, com informações do Banco Mundial (Banco Mundial, 2007), que o teto de recursos para essas transferências ou pagamentos é redefinido anualmente, com base em critérios como a série histórica de produção e de pagamentos, parâmetros técnicos (como internação/habitante por ano) e metas fixadas para programas específicos.

O fundo de Atenção de Média e Alta Complexidade agrega recursos a serem destinados a procedimentos ambulatoriais e hospitalares mais sofisticados e que exigem maior tecnologia e especialização, tais como: cirurgias, fisioterapia e exames mais complexos para diagnósticos. Essas transferências também são obrigatórias e condicionais, baseadas na produção e submetidas a um teto de recursos. O Ministério da Saúde também realiza um aporte adicional de recursos a estados e municípios capacitados a oferecer atendimentos de alta complexidade, para onde são encaminhados pacientes de todo o país (Mendes, Miranda e Cossio, 2008).

A modalidade Vigilância em Saúde é composta pelos blocos Vigilância e Promoção da Saúde e Vigilância Sanitária. Já a Assistência Farmacêutica engloba os componentes Básico e Estratégico da Assistência Farmacêutica, além de Medicamentos de Dispensação Excepcional (Brasil, 2010). Ambas as modalidades contam com um componente básico, financiado através de recursos per capita, além de outros componentes financiados de acordo com a cobertura e/ ou produção dos programas até um limite estabelecido por unidade subnacional. 
O fundo Gestão do SUS recebe recursos relacionados à Qualificação da Gestão do SUS e Implantação de Ações e Serviços de Saúde. Os recursos devem ser destinados a ações de regulação e controle, planejamento e orçamento, gestão do trabalho, educação em saúde, entre outros. O fundo também recebe recursos para a implantação de novas unidades de saúde de algumas categorias específicas, tais como o Centro de Especialidades Odontológicas (CEO) e o Serviço de Atendimento Móvel de Urgência (SAMU). Existem critérios específicos de valores a serem transferidos associados a cada tipo de ação, novamente com tetos delimitados (Brasil, 2012).

Após o Pacto pela Saúde, os estados e municípios passaram também a poder receber recursos através de um fundo destinado a investimentos adicionais (através do bloco Investimentos), que são liberados mediante a aprovação de projetos específicos pelo Ministério da Saúde. Esses projetos devem ser submetidos à Comissão Intergestores Bipartite (CIB), que avalia sua conformidade com os planos estaduais e municipais. Os projetos aprovados são formalizados em portaria do Ministério da Saúde, com o valor, o período de execução e o cronograma de desembolso dos recursos financeiros a serem transferidos (Brasil, 2012).

Existe ainda a possibilidade de que os estados e municípios estabeleçam Convênios com o governo federal para a realização de ações e programas de responsabilidade mútua. Nesses casos, o governo federal também transfere recursos para o ente conveniado e pode exigir algum tipo de contrapartida financeira, a depender do que foi acordado. Além dos estados e municípios, entidades filantrópicas e organizações não governamentais também podem estabelecer Convênios com o Ministério da Saúde para o financiamento de projetos específicos na área de saúde. Os repasses através de Convênios são transferências voluntárias e, segundo dados da Cartilha do Ministério da Saúde (Brasil, 2006), representavam menos de 10\% do montante transferido em 2006.

Conforme podemos perceber, todas as transferências e aportes de recursos realizados pelo Ministério da Saúde aos estados e municípios são condicionais. Isto é, precisam ser necessariamente aplicados em propósitos específicos na área da saúde, embora haja flexibilidade entre as ações que se encaixam em cada bloco de financiamento. Além disso, com exceção das transferências para o bloco Investimentos, 
todas as demais transferências fundo a fundo são obrigatórias. Isto é, ocorrem de forma regular e automática, sem a necessidade de aprovação prévia, e seu valor é baseado em algum critério per capita ou de produção e/ou cobertura dos programas a que se destina. As transferências para o bloco de Investimentos, além dos recursos recebidos através de Convênios, são voluntárias. Isto é, só ocorrem mediante aprovação de projeto específico pelo Ministério da Saúde.

É importante salientar que, estritamente de acordo com as regras, apenas os recursos recebidos através dos Convênios, que são a minoria, podem exigir algum tipo de contrapartida financeira do ente que os recebe. Ou seja, a priori, e em conformidade com os princípios de redistribuição e equidade a que se propõe o SUS, não deveria haver uma relação positiva entre capacidade dos estados e municípios em arcar com as despesas e/ou investimentos em saúde e as transferências recebidas do governo federal. Este trabalho estuda empiricamente essa última relação citada.

\section{Transferências intergovernamentais: Aspectos teóricos, equida- de e flypaper effect}

Mendes, Miranda e Cossio (Mendes, Miranda e Cossio, 2008) analisam a estrutura das transferências intergovernamentais no Brasil, procurando identificar se ela atende aos objetivos a que se propõe e trazendo sugestões de aperfeiçoamento. Os autores destacam um conjunto de características desejáveis a um sistema de transferências, as quais, no entanto, podem ser total ou parcialmente conflitantes.

Oito características são apontadas como desejáveis a um sistema de transferências: (i) autonomia subnacional, que se refere à autonomia dos governos locais para gerir seus recursos e fazer escolhas quanto à sua alocação (a literatura argumenta que os governos locais, por estarem mais próximos aos cidadãos, têm melhores condições de priorizar as políticas de acordo com as suas necessidades, bem como de escolher a melhor forma de implementá-las); (ii) accountability, que se refere ao grau em que os eleitores conseguem acompanhar e monitorar o recebimento e a aplicação dos recursos pelo governo local; (iii) redistribuição regional, de modo que o mecanismo de transferências possa ser utilizado para auxiliar na correção de desigualdades 
sociais e econômicas que se manifestam entre as regiões; (iv) redução do hiato fiscal, que corresponde à busca pela redução da "diferença entre o custo do conjunto de bens e serviços públicos necessários e economicamente viáveis em determinada região e a capacidade local de financiamento dessa despesa para um dado custo marginal de financiamento"; (v) flexibilidade para absorção de choques, que se refere à flexibilidade e agilidade do sistema de redirecionar recursos para necessidades específicas quando surgem situações imprevistas (choques); (vi) internalização das externalidades, que se refere à capacidade do sistema de equacionar de modo eficiente (ou internalizar) as externalidades inerentes ao serviço prestado (pode ser de interesse nacional que uma localidade mais desenvolvida forneça determinado serviço a uma população maior do que a sua própria, gerando uma externalidade positiva para outras localidades); (vii) independência de fatores políticos e responsabilidade fiscal, pois é desejável que as regras sejam independentes de fatores políticos e que mantenham o interesse dos governos locais em explorar suas próprias bases tributárias; e, finalmente, (viii) incentivo à gestão eficiente, que se refere à capacidade do sistema de aumentar os valores recebidos por aqueles governos que demonstrem melhor desempenho, gerando incentivos a uma boa gestão.

Mendes, Miranda e Cossio discutem os prós e contras das transferências do SUS à luz das características desejáveis por eles apontadas. Como ponto positivo, ressaltam a independência de fatores políticos, especialmente para as modalidades fundo a fundo, uma vez que as regras são preestabelecidas e têm se tornado cada vez mais transparentes. Comentam ainda que o ponto não é tão válido para a modalidade de convênios, que hoje representa a minoria dos recursos transferidos.

Com relação à autonomia subnacional, algo fortemente presente nos princípios que nortearam a criação SUS, os autores comentam que ela ocorre de forma parcial, pois, embora as transferências per capita devam ser aplicadas no bloco a que se destinam, os governos locais possuem liberdade para escolher a melhor alocação dentro das ações e programas previstos em cada bloco. Além disso, a adesão a programas específicos que são financiados acontece segundo escolha dos próprios governos locais, desde que discutidos e aprovados pelos respectivos Conselhos de Saúde. Os autores comentam, ainda, que o SUS apresenta bons resultados na questão da redistribuição de 
recursos ao longo do território. Por outro lado, como as transferências do SUS são obrigatórias e pró-cíclicas, uma vez que estão diretamente relacionadas ao crescimento do PIB e da arrecadação anual dos governos locais, há baixa flexibilidade para absorção de choques.

Para Mendes, Miranda e Cossio, o grande problema no processo de transferências do SUS é a sua incapacidade de distribuir os recursos segundo critérios de necessidade de saúde, como também de premiar bons resultados. Aparentemente, não há incentivos a uma gestão eficiente de recursos. A Lei Orgânica da Saúde, de 1990, previa inicialmente critérios de transferências baseados no perfil demográfico e epidemiológico de cada região, além de características quantitativas e qualitativas da rede de saúde e desempenho no período anterior. Entretanto, a falta de acordo político em torno desses critérios impossibilitou sua aplicação na prática. Uma consequência desse fenômeno é o fato de que, embora os recursos estejam hoje mais homogeneamente distribuídos ao longo do terrítório, os indicadores de saúde estão convergindo para patamares diferentes entre as regiões do país.

A análise empírica realizada neste trabalho teve foco no aspecto redistributivo das transferências do SUS. Além disso, embora não seja o tópico principal, ajuda a discutir algumas das outras características desejáveis apontadas acima, como a independência de fatores políticos e a relação do volume transferido com as necessidades de saúde de cada localidade. Em particular, nossa análise empírica traz evidências de que, apesar dos bons resultados do SUS com relação à redistribuição de recursos ao longo do território comparativamente ao período anterior à implantação do sistema, ainda é possível que os municípios mais ricos estejam se beneficiando de montantes relativamente maiores de transferências per capita. A priori, a principal evidência nesse sentido é a correlação positiva e significante entre a receita municipal (receita cujas fontes foram detalhadas na seção anterior deste artigo) e o recebimento de transferências para a saúde por parte dos municípios.

Uma análise das transferências de recursos do governo federal para os municípios nos mostra que existe uma dispersão relativamente grande dos volumes transferidos quando analisados em uma base per capita, mesmo quando nos restringimos a regiões específicas. Utilizando dados do SIOPS/Ministério da Saúde e considerando ape- 
nas os recursos transferidos através das modalidades fundo a fundo, observamos que o coeficiente de variação $(\mathrm{CV}){ }^{1}$ dos recursos per capita do SUS recebidos pelos municípios brasileiros é igual a 46\%, sendo menor na Região Nordeste (CV de 39\%) e maior na Região Sudeste (CV de 56\%). Além disso, conforme já foi destacado, observa-se uma correlação positiva entre o volume recebido em transferências fundo a fundo para a saúde por município e a receita municipal, ambos em uma base per capita, sendo que essa correlação persiste mesmo quando analisamos separadamente cada uma das regiões brasileiras.

A Tabela 1, a seguir, apresenta o coeficiente de correlação de Pearson entre essas variáveis por região, além da correlação entre o gasto per capita de recursos próprios em saúde e as transferências fundo a fundo do SUS, usando dados referentes aos municípios brasileiros no período de 2002 a 2010. Para comparabilidade dos valores monetários, todos eles foram corrigidos com o IPCA para valores na mesma referência. ${ }^{2}$ Todas as correlações foram significantes a $1 \%$.

Tabela 1 - Correlação das transferências da modalidade fundo a fundo do SUS com o gasto de recursos próprios em saúde e com a receita municipal, no período de 2002 a 2010 (base per capita)

\begin{tabular}{|c|c|c|}
\hline Região & Gastos próprios em saúde & Receita Municipal \\
\hline Centro-Oeste & $24,60 \%$ & $21,70 \%$ \\
\hline Nordeste & $14,40 \%$ & $12,70 \%$ \\
\hline Norte & $34,20 \%$ & $33,50 \%$ \\
\hline Sudeste & $19,20 \%$ & $18,90 \%$ \\
\hline Sul & $32,20 \%$ & $30,60 \%$ \\
\hline Brasil & $16,10 \%$ & $14,90 \%$ \\
\hline
\end{tabular}

Fonte: SIOPS/Ministério da Saúde. Elaboração própria.

Em princípio, tais relações não seriam esperadas. O que se espera, no mínimo em decorrência das exigências legais, é uma correlação positiva entre receita municipal per capita e gastos locais per capita em saúde. Contudo, como estamos nos restringindo às transferências

${ }^{1}$ O coeficiente de variação (CV) de uma distribuição é dado pela razão entre seu desvio padrão e sua média. A medida auxilia na mensuração da homogeneidade de um conjunto de dados.

2 Conforme seção 4, tópico 4.1 .

Estud. Econ., São Paulo, vol.45, n.1, p. 33-63, jan.-mar. 2015 
da modalidade fundo a fundo do SUS, o recebimento dessas transferências para a saúde não exige explicitamente uma contrapartida financeira de gasto de recursos locais, logo não há motivo para esperar uma correlação positiva entre as transferências fundo a fundo do SUS e a receita municipal.

A análise empírica empreendida neste estudo explora e investiga essa relação positiva. A depender dos motivos que levem a essa relação, não necessariamente ela seria desejada, uma vez que os municípios que arrecadam mais, em princípio, têm mais condições de arcar com as necessidades de saúde de sua população.

\subsection{Transferências condicionais, Flypaper effecte o modelo de Chernick}

Um tópico bastante explorado na literatura que trata das transferências intergovernamentais é um fenômeno que ficou conhecido como o "flypaper effect". Tal fenômeno, em princípio, contradiz uma previsão teórica simples. Se a decisão do governo local reflete as preferências do eleitor mediano, as tranferências incondicionais (lumpsum) para governos locais deveriam ser gastas do mesmo modo que qualquer outro aumento de renda. Em particular, seria possível que se observasse uma redução de impostos locais, uma vez que, em determinadas situações, nem todo aumento de renda deveria ser gasto em bens públicos.

Entretanto, conforme descrevem Hines e Thaler (Hines e Thaler, 1995), diversos estudos investigaram os efeitos de vários tipos de tranferências para governos locais e a grande maioria deles concluiu que o gasto público local é muito mais estimulado quando ocorrem transferências do que seria esperado pela teoria. Essa observação empírica ficou conhecida como o flypaper effect, pois o dinheiro tende a ser gasto pela jurisdição local que o recebe. ${ }^{3}$

Mesmo quando tratamos de transferências condicionais, como é o caso das transferências na área da saúde no Brasil, Hines e

3 Segundo Hines e Thaler [Hines e Thaler 1995], essa nomenclatura foi atribuída ao fenômeno por Arthur Okun, uma vez que o dinheiro "gruda" na jurisdição que o recebe: "money sticks where it hits". 
Thaler (Hines e Thaler, 1995) comentam que sempre é possível derivar uma previsão baseada na teoria econômica ortodoxa a respeito da relação entre essas transferências e os gastos e arrecadação locais. Muitos desses modelos teóricos preveem que um aumento das transferências condicionais, direcionadas a um determinado setor de serviços públicos, poderia causar, dependendo das regras e das restrições de gasto, uma queda dos impostos e do gasto de recursos locais, mas essas previsões em geral são refutadas pelas análises empíricas.

Chernick (Chernick, 1979) e Knight (Knight, 2002) apresentam modelos que buscam explicar casos (não previstos pela teoria ortodoxa) de relação positiva entre gastos locais e transferências condicionais. Chernick investiga transferências que são feitas com base em projetos (project grants), a partir de informações sobre transferências para o setor de saneamento do Departamento de Construção Civil e Desenvolvimento Urbano dos Estados Unidos. Knight estuda o caso de transferências condicionais (closed-end matching grants) feitas pelo governo federal para os estados para a construção e manutenção de estradas.

O modelo de Chernick (Chernick, 1979) conclui que o volume de transferências recebido localmente depende da propensão local a "pagar" pelas transferências, isto é, da propensão local a contribuir com recursos locais para cada quantidade recebida de transferências. Nesse modelo, o governo central procura maximizar o produto induzido por cada unidade monetária despendida e, por isso, prefere transferir para as localidades que contribuem mais com recursos locais. Ou seja, o governo central, através de seu processo de transferências, induz à relação entre gastos e transferências.

Knight (Knight, 2002), por sua vez, constrói um modelo de barganha para a distribuição das transferências entre estados, em que um comitê com um representante de cada estado determina a distribuição das transferências entre estados. Assume-se a existência de dois tipos de estado: com elevada e baixa preferência por bens públicos. Como a proposta de distribuição de transferências só é aprovada por maioria e o chefe do comitê quer maximizar as transferências para o seu estado, este faz uma aliança com os estados que mais valorizam os bens públicos e a distribuição de transferências é aprovada de tal modo que os estados que mais valorizam os bens públicos recebem mais. 
O modelo de Chernick (Chernick, 1979) se aproxima mais do objeto de interesse deste estudo, uma vez que a distribuição dos recursos do SUS para os municípios não se baseia em uma eleição feita em um comitê representativo. Além disso, Chernick desenvolve um modelo empírico para investigar a relação de causalidade entre a propensão local a gastar recursos próprios e o recebimento de transferêncas condicionais. Apesar de uma parte das transferências fundo a fundo do SUS ser determinada por critérios de equalização, com valores per capita, uma boa parte é determinada a partir da adesão a programas e demonstração de prestação de serviços por parte dos municípios. É possível que os municípios com maior "propensão local a complementar as transferências", determinada pela maior disponibilidade de recursos locais, sejam os que mais aderem aos programas do SUS e, com isso, terminem por receber mais recursos do governo federal.

Especificamente para o caso brasileiro, muitos trabalhos buscam evidenciar ou explicar o flypaper effect nas transferências do governo federal para os estados e municípios. A maioria dos estudos encontrados tratam, entretanto, das transferências intergovernamentais incondicionais, como os de Cossio e Carvalho (Cossio e Carvalho, 2001), Sakurai (Sakurai, 2009), Marconi, Arvate, Moura Neto e Palombo (Marconi et al., 2009) e Mattos, Rocha e Arvate (Mattos, Rocha e Arvate, 2011). Quando se trata especificamente das transferências governamentais na área da saúde, a maioria dos trabalhos encontrados concentra-se mais em medir a eficiência dessas transferências através da observação de seu impacto em determinados indicadores. Varela, Martins e Fávaro (Varela, Martins e Fávero, 2010), por exemplo, utilizam a técnica do DEA - Data Envelopment Analysis - e concluem, dentre outras coisas, que seria possível aumentar a quantidade de serviços prestados à população sem a necessidade de que os municípios obtivessem recursos adicionais. Exceções são os estudos de Peixoto, Rocha, Nishijima e Postali (Peixoto, Rocha, Nishijima e Postali, 2012) e de Parmagnani e Rocha (Parmagnani e Rocha, 2013). O primeiro usa as transferências do SUS para medir descentralização no financiamento da saúde e estudar a relação entre descentralização e corrupção, não encontrando correlação estatisticamente significativa. O segundo avalia o impacto das transferências do SUS sobre os gastos em saúde, procurando verificar se a maior parte dos recursos transferidos pelo governo está sendo utilizada para serviços de saúde ou para serviços outros que não a saúde a nível local. 
O estudo encontra evidências de que nem todo recurso do SUS é aplicado em saúde (efeito fungibilidade)."

\section{Estratégia empírica e dados}

O objetivo principal da análise empírica deste trabalho é estudar em que medida a receita municipal afeta o volume de transferências fundo a fundo do SUS recebido por cada município. Foram estimadas regressões em painel com efeitos fixos que buscam identificar os determinantes das transferências condicionais fundo a fundo na área da saúde. O modelo empírico estimado baseia-se em parte no modelo empírico apresentado por Chernick (Chernick, 1979), porém, como será visto, as hipóteses que estão por trás do modelo proposto neste estudo são diferentes.

Antes de apresentar os detalhes da regressão estimada, é importante destacar que o uso do modelo de efeitos fixos controla, em grande parte, uma série de potenciais problemas de omissão de variáveis. Por exemplo, uma possível explicação para a correlação observada entre receita municipal e transferências fundo a fundo do SUS é a de que os municípios com maior receita municipal per capita sejam também aqueles que fornecem serviços mais especializados e de referência para uma população maior do que a sua, que tendem a ser os municípios maiores. O SUS prevê transferências adicionais para esses centros de referência, que fornecem serviços de alta complexidade. O modelo de efeitos fixos, ao controlar todas as características municipais fixas no tempo, controla em grande parte esse efeito. Além disso, variáveis como tamanho da população, média da população de municípios vizinhos, população urbana e renda per capita são incluídas na regressão. Poder-se-ia imaginar também que a própria demanda por serviços de saúde da população local fosse maior nos municípios com maior arrecadação média ao longo do tempo. Porém, assumindo que as preferências são fixas no período de tempo observado, e controlando os outros potenciais determinantes da demanda por transferências, podemos admitir que esse efeito também é controlado. 
A partir da análise das regras que definem as transferências do SUS, formulou-se neste estudo uma outra hipótese, para a qual buscamos evidências empíricas. Como já foi ressaltado, os recursos repassados fundo a fundo pelo SUS são divididos basicamente em dois tipos: as transferências com base per capita e as transferências com base em nível de produção e/ou cobertura de programas e ações de saúde.

Quando se trata das transferências que dependem de produção e/ ou cobertura, há sempre um valor estabelecido para o aporte do governo central por unidade de produto. Pode-se conjecturar, portanto, que o financiamento do governo federal a esses programas é parcial, podendo ser insuficiente. Dependendo da forma como esses valores estejam sendo calculados, é possível que os recursos do governo federal não cubram parte suficiente dos gastos e, nesse caso, recursos locais precisariam ser investidos. Se os repasses do SUS estiverem exigindo de forma implícita essa contrapartida em gastos locais, alguns municípios podem ter mais incentivos do que outros para aderir a determinados programas ou ações.

Assim, nossa hipótese é a de que os municípios que mais arrecadam, por terem mais recursos que devem ser investidos em saúde, são os que têm mais incentivos a aderir ou ofertar os programas e serviços que recebem repasses do governo federal através do SUS. Para tais municípios, o "custo" de complementar os recursos da União e arcar com as despesas totais dos programas de saúde seria menor. Ou ainda, pode-se admitir que há um custo de aderir aos programas se os requisitos básicos exigidos não forem atendidos, o que, por sua vez, está relacionado à disponibilidade de recursos locais. $\mathrm{O}$ custo de aderir aos programas e não cumprir com os requisitos básicos estaria ligado à prestação de contas e ao monitoramento, mesmo que estes sejam feitos de forma precária. ${ }^{4}$

Enfim, a ideia subjacente é que o próprio mecanismo de transferências do SUS pode induzir, de forma implícita, os governos locais com maior receita a aderir a mais programas e, portanto, a receber mais transferências.

\footnotetext{
4 A prestação de contas é feita a partir dos relatórios gestão, que são submetidos à aprovação do conselho municipal de saúde e encaminhados ao Ministério da Saúde, à Secretaria de Saúde e ao tribunal de contas a que o município estiver jurisdicionado. O monitoramento é feito pelos órgãos de controle interno e externo.
} 


\subsection{O modelo empírico}

Conforme já mencionado, Chernick (Chernick, 1979) estudou transferências no setor de saneamento nos Estados Unidos, que eram concedidas de acordo com a aprovação de projetos submetidos ao órgão central. Após a apresentação de seu modelo teórico, Chernick propõe um modelo para teste empírico em duas etapas. A primeira etapa é a de escolha, por parte do poder central, dos projetos a serem financiados; e a segunda é a determinação do volume de transferências destinado a cada projeto (que, para Chernick, depende da propensão local a contribuir, mesmo que essa contribuição não seja obrigatória). No caso das transferências fundo a fundo do SUS, não há municípios que não recebam esses recursos. Logo, a estimação em duas etapas proposta por Chernick não se aplica. Neste estudo, a ideia seria estimar apenas uma segunda etapa, isto é, uma equação de determinação do volume per capita de transferências fundo a fundo do SUS destinado a cada município.

A equação geral estimada neste estudo tem a seguinte especificação:

$\log \_$transfSUS_hab $_{i t}=\beta_{1} \log _{-}$ReceitaMunicipal_hab $b_{i t}+\boldsymbol{\theta} \mathbf{X}_{i t}+\gamma \mathbf{D}_{t}+\alpha_{i}+\varepsilon_{i t}$

onde $\log _{\text {_transfSUS_hab }}$ it corresponte ao logaritmo do volume total per capita de transferências fundo a fundo recebidas pelo município i no ano t (sem a modalidade de convênios, explicada na seção 2, e já excluídos os valores per capita recebidos através do PAB fixo, os quais não dependem da adesão a programas específicos); log_ReceitaMunicipal_hab ${ }_{i t}$ corresponde ao logarítmo da receita municipal per capita (fontes detalhadas na seção 2 deste artigo) do município i no ano t; $\boldsymbol{X}_{\mathrm{it}}$ corresponde a um vetor de variáveis de controle; $\mathbf{D}_{\mathrm{t}}$ corresponde a um vetor de variáveis binárias de ano; e $\alpha_{\mathrm{i}}$ corresponde ao efeito fixo municipal não observado.

Queremos testar a hipótese de que, em (1), $\beta_{1}$ seja positivo e significante, mesmo quando variamos alguns controles. Isto é, nosso objetivo é testar se choques positivos na receita municipal levam também a aumentos nas transferências fundo a fundo recebidas do SUS, já descontado o montante referente ao PAB fixo. A equação também foi estimada separando a receita municipal per capita em dois componentes: arrecadação local de impostos e total de transferências 
incondicionais recebidas (não ligadas à saúde e calculadas com base em regras fixas e preestabelecidas, como o Fundo de Participação dos Municípios (FPM)).

A estimação pelo modelo de efeitos fixos permite controlar o fato de que as variáveis observadas, como a própria arrecadação municipal de impostos, podem ser correlacionadas com as preferências dos cidadãos, no caso representadas em $\alpha_{i}$. Para eliminar o efeito de escala, todos os valores utilizados no estudo foram considerados em uma base per capita. Além disso, optou-se por trabalhar com os valores monetários transformados em logaritmo, para reduzir o efeito de outliers como também para que se obtivesse uma interpretação dos coeficientes em termos de elasticidades. Como as regressões também envolvem valores monetários no tempo, optou-se ainda por corrigi -los através do IPCA, ${ }^{5}$ de modo a torná-los valores referenciados no ano 2011. Para efetuar a correção, utilizou-se a variação percentual acumulada do indicador entre dezembro do ano em que o valor está referenciado e dezembro de 2011.

Um ponto importante é que, no modelo empírico, a receita municipal foi considerada uma variável exógena. Não há motivos para acreditar, a princípio, que fatores não observados variantes no tempo influenciem ao mesmo tempo a receita municipal e o recebimento das transferências fundo a fundo do SUS. No que se refere ao componente de transferências incondicionais da arrecadação local, é muito importante notar que essas transferências incondicionais (não vinculadas à saúde) são, em geral, calculadas de acordo com regras fixas que dependem predominantemente do tamanho da população e do PIB municipal, estadual e nacional, todas variáveis controladas na regressão. Além disso, a arrecadação de impostos do município depende fundamentalmente do nível de atividade econômica local, que também é controlada na regressão (a correlação entre PIB municipal e arrecadação per capita é da ordem de 60\%).

Segundo Mattos, Rocha e Arvate (Mattos, Rocha e Arvate, 2011), existem evidências de que um aumento no volume total de transferências incondicionais poderia incentivar os municípios a reduzirem os esforços despendidos para a arrecadação de tributos. $\mathrm{O}$ trabalho citado, entretanto, não é específico para o setor de saúde. Isto é, por

O IPCA - Índice Nacional de Preços ao Consumidor Amplo, calculado pelo IBGE, é o índice oficial de inflação do Brasil. 
mais que um aumento das transferências incondicionais possa reduzir os esforços de arrecadação local, é muito pouco provável que esse relaxamento no esforço de arrecadação local aconteça no caso de um aumento das transferências do SUS, direcionadas especificamente para o setor de saúde. ${ }^{6}$ Em suma, existem fortes razões para aceitar a exogeneidade da variável receita municipal na equação proposta, porém, por rigor, em geral manteremos neste artigo o uso do termo correlação.

Os controles utilizados em cada estimação nos ajudarão a identificar o efeito marginal de variações na receita municipal sobre o recebimento de transferências do SUS. Ademais, o impacto obtido para alguns controles também será importante para entender características e consequências do processo de transferências de recursos no SUS. Os controles municipais buscam captar fatores de oferta e de demanda por serviços de saúde, além de fatores políticos, e podem ser divididos nos seguintes grupos: (i) renda e população (inclui variáveis demográficas), (ii) oferta de serviços de saúde, (iii) necessidades de saúde e (iv) fatores políticos. A princípio, fatores políticos não deveriam influenciar a adesão aos programas e ações de saúde vinculados aos Pisos variáveis. Contudo, decidiu-se controlar fatores políticos para investigar esse tema.

\subsection{Os dados}

Os dados referentes às transferências do SUS foram obtidos através do Sistema de Informações sobre Orçamentos Públicos em Saúde (SIOPS) do Ministério da Saúde. O SIOPS é composto por um banco de dados que é alimentado pelos próprios estados e municípios e tem por objetivo apurar as receitas totais e os gastos em ações e serviços públicos de saúde. O preenchimento do SIOPS deve estar de acordo com as informações contábeis geradas e mantidas pelos estados e municípios. Os dados referentes à população de cada município, utilizados para computar os valores per capita, foram obtidos do Departamento de Atenção Básica do Ministério da Saúde (DATASUS). A informação refere-se à população estimada

6 Além disso, de acordo com o argumento desses autores esperar-se-ia uma correlação negativa entre arrecadação local e transferências, ao contrário daquilo que se espera neste estudo.

Estud. Econ., São Paulo, vol.45, n.1, p. 33-63, jan.-mar. 2015 
pelo Instituto Brasileiro de Geografia e Estatística (IBGE) para o Tribunal de Contas da União (TCU), que é utilizada pelo Ministério da Saúde para os repasses do Piso de Atenção Básica (PAB).

Adicionalmente, através da Secretaria do Tesouro Nacional (STN), obtiveram-se dados desagregados de receita municipal, para que fosse possível separar os efeitos associados à arrecadação de impostos e às transferências incondicionais.

A Tabela 2 apresenta a descrição e a fonte de todas as variáveis utilizadas neste estudo. Todas elas, com exceção das variáveis de PIB, foram obtidas para o período de 2002 a 2010. O PIB municipal e seus demais agrupamentos estão disponíveis somente até 2009. Todas as variáveis são expressas em valores ou quantidades anuais para cada município. Além disso, no caso das variáveis monetárias, antes de aplicar o log, os valores foram corrigidos pelo IPCA para valores em R\$ de 2011.

Em cada ano de referência entre 2002 e 2010, todos os municípios que apresentavam informações de receita municipal e de transferências SUS preenchidas e válidas tanto no SIOPS quanto na STN foram considerados na base do estudo. Classificamos como válidas as informações monetárias não inferiores a $\mathrm{R} \$ 1,00$. Apesar das exclusões, a base construída cobre pelo menos 92\% dos municípios brasileiros em cada ano de referência, sendo que, em 2006, cobre 97,2\% dos municípios (5410 dos 5563 municípios). Em sua maioria, as observações foram excluídas por ausência de informação em uma das fontes. 


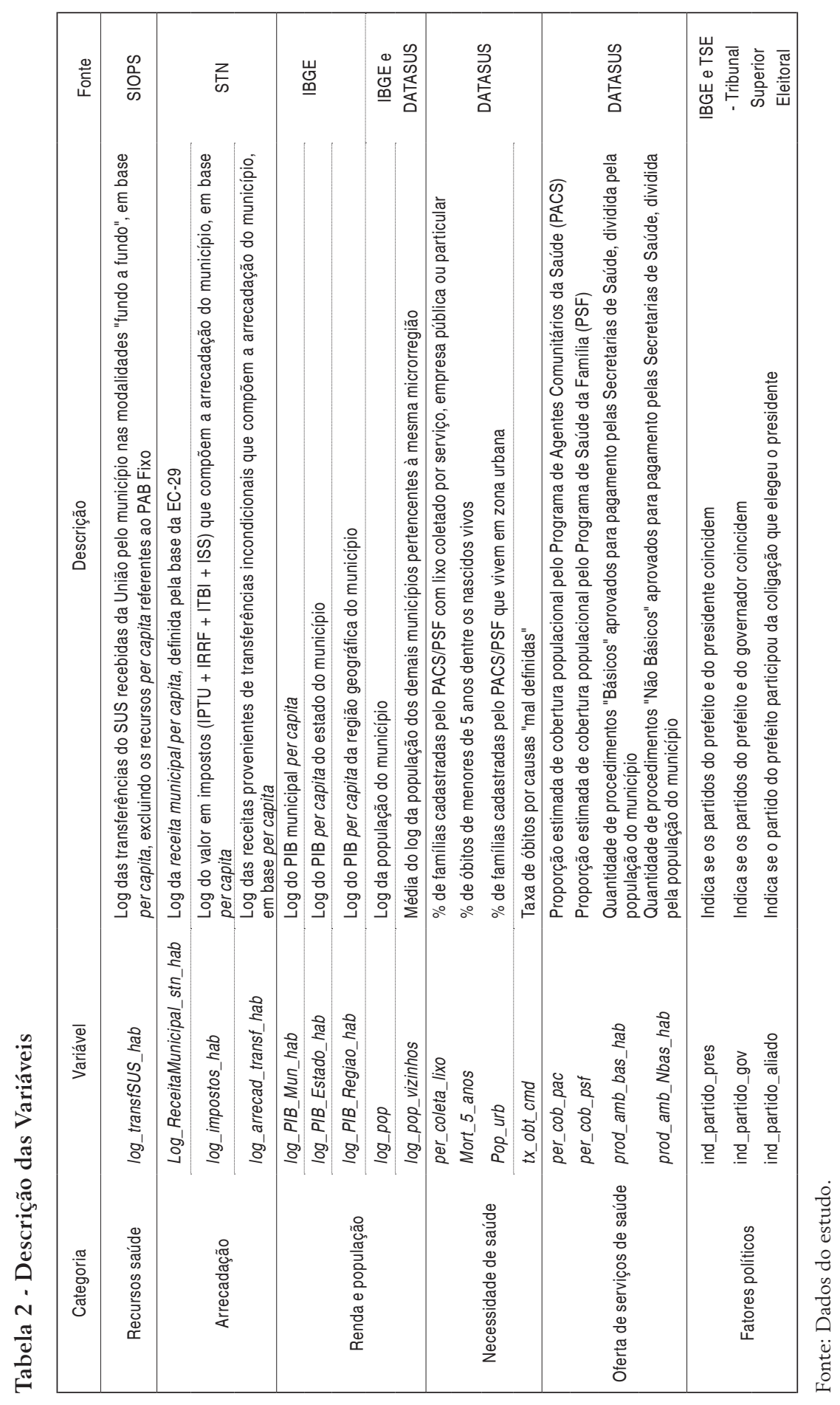

Estud. Econ., São Paulo, vol.45, n.1, p. 33-63, jan.-mar. 2015 
Por fim, a Tabela 3 traz estatísticas descritivas de todas as variáveis utilizadas nas regressões em painel estimadas. Como já comentado, para efeito das regressões considerou-se o logaritmo dos valores monetários. A tabela mostra que os dados são, em geral, bem comportados, não apresentando muitos outliers. A própria transformação logarítmica auxilia também nessa questão com a redução da escala.

Tabela 3- Estatísticas descritivas das variáveis utilizadas nas regressões em painel

\begin{tabular}{|c|c|c|c|c|c|}
\hline Variável & $\% p^{*}$ & média & desvio & $\min$ & $\max$ \\
\hline log_transfSUS_hab & $100 \%$ & 4,49 & 0,54 & 1,09 & 6,81 \\
\hline log_ReceitaMunicipal_stn_hab & $100 \%$ & 6,85 & 0,57 & 4,4 & 9,62 \\
\hline log_impostos_hab & $100 \%$ & 3,96 & 0,93 & $-3,47$ & 8,18 \\
\hline log_arrecad_transf_hab & $100 \%$ & 6,73 & 0,58 & 3,02 & 9,45 \\
\hline log_PIB_Mun_hab & $89 \%$ & 9,01 & 0,73 & 7,24 & 12,93 \\
\hline log_PIB_Estado_hab & $89 \%$ & 9,49 & 0,48 & 8,37 & 10,3 \\
\hline log_PIB_Regiao_hab & $89 \%$ & 9,58 & 0,47 & 8,8 & 10,15 \\
\hline $\log \_p o p$ & $100 \%$ & 9,39 & 1,15 & 6,68 & 16,22 \\
\hline log_pop_vizinhos & $98,80 \%$ & 10,55 & 1,55 & 8,5 & 25,42 \\
\hline per_cob_pac & $100 \%$ & 83,71 & 29,84 & 0 & 100 \\
\hline per_cob_psf & $100 \%$ & 70,52 & 35,88 & 0 & 100 \\
\hline prod_amb_bas_hab & $99 \%$ & 9,46 & 10,56 & 0 & 557,92 \\
\hline prod_amb_Nbas_hab & $100 \%$ & 2,28 & 3,28 & 0 & 96,17 \\
\hline Mort_5_anos & $89 \%$ & 2,08 & 1,75 & 0 & 42,86 \\
\hline Pop_urb & $89 \%$ & 64,58 & 33,83 & 0 & 100 \\
\hline per_coleta_lixo & $95 \%$ & 61,55 & 26,73 & 0 & 100 \\
\hline$t x \_o b t \_c m d$ & $100 \%$ & 13,72 & 15,45 & 0 & 100 \\
\hline ind_partido_pres & $100 \%$ & 0,09 & 0,28 & 0 & 1 \\
\hline ind_partido_gov & $95,50 \%$ & 0,34 & 0,47 & 0 & 1 \\
\hline ind_partido_aliado & $100 \%$ & 0,10 & 0,30 & 0 & 1 \\
\hline
\end{tabular}

*\%p: \% de preenchimento da variável.

Fonte: Dados do estudo. 


\section{Resultados}

Com o objetivo de interpretar melhor os resultados, foram estimadas seis regressões em painel com efeitos fixos, todas com especificação semelhante à apresentada na Equação (1). A cada estimação, novos controles foram adicionados. A variável resposta utilizada foi sempre o logaritmo das transferências SUS per capita recebidas pelos municípios. Como já mencionado, para a composição da variável resposta, considerou-se apenas as transferências fundo a fundo e descontou-se os valores provenientes do PAB fixo, que são valores per capita transferidos aos municípios independentemente da adesão aos programas de saúde. As dummies de ano, para controlar eventuais tendências no tempo, foram mantidas em todas as regressões. Os resultados evidenciam que a arrecadação municipal é de fato um fator que influencia positivamente o recebimento de transferências fundo a fundo do SUS. Os coeficientes associados aos dois tipos de arrecadação municipal se mantiveram positivos e significantes a $1 \%$ em todas as regressões estimadas (com a exceção de que as transferências incondicionais foram significantes a $2 \%$ na regressão com todos os controles).

A Tabela 4 apresenta os coeficientes estimados para diferentes especificações. A Equação (1) com variável de interesse desagregada (em que a receita municipal foi separada em dois componentes) foi estimada a partir de cinco especificações, primeiramente apenas com as duas variáveis de interesse, arrecadação local de impostos e arrecadação local de transferências incondicionais, além das variáveis binárias de ano. Para as demais regressões, foram sendo acrescentadas as seguintes variáveis de controle: [ii] controles de renda e tamanho da população; [iii] controles de oferta em saúde; [iv] indicadores de necessidade de saúde; e [v] fatores políticos. Adicionalmente, estimou-se a regressão [vi], que corresponde à Equação (1) sem a variável de interesse desagregada, em que foi considerada a receita municipal como um todo (composta predominantemente pela soma da arrecadação local de impostos e das transferências incondicionais), juntamente com todas as variáveis de controle. ${ }^{7}$

7 As regressões também foram estimadas admitindo correlação espacial e os resultados não se alteram, sendo os coeficientes das variáveis de interresse apenas um pouco maiores.

Estud. Econ., São Paulo, vol.45, n.1, p. 33-63, jan.-mar. 2015 
No caso das variáveis monetárias, uma vez que todas estão na forma logarítmica, assim como a variável dependente, o coeficiente estimado pode ser interpretado diretamente como uma elasticidade. Por exemplo, pela regressão [i], temos que, em média e sem mais controles, um aumento de $1 \%$ na arrecadação municipal per capita de transferências incondicionais traz um aumento de aproximadamente $0,27 \%$ no montante recebido em transferências fundo a fundo SUS. Todas as variáveis explicativas não monetárias, com exceção das produções ambulatoriais e dos indicadores de partido do prefeito, têm sua unidade expressa em pontos percentuais. Ou seja, seu coeficiente representa o impacto percentual nas transferências SUS de uma variação de 1 ponto percentual na respectiva variável, tudo mais constante.

Mesmo após a inclusão de todos os controles, temos que variações de $1 \%$ no total arrecadado de transferências incondicionais e de impostos locais ainda acarretam uma variação de aproximadamente $0,1 \%$ no volume per capita recebido de transferências fundo a fundo do SUS. ${ }^{8}$

8 Substituindo a variável receita municipal pela variável gastos em saúde usando recursos locais instrumentalizada, o coeficiente também é positivo, significante e de aproximadamente 0,16 . 
Tabela 4 - Regressões em painel com efeitos fixos - Variável dependente: log_transfSUS_hab

\begin{tabular}{|c|c|c|c|c|c|c|c|}
\hline & & & & Coeficientes & e p-valores & & \\
\hline & Varıavel & [i] & [ii] & [iii] & [iv] & {$[\mathrm{v}]$} & [vi] \\
\hline $\begin{array}{l}\text { Arrecadação } \\
\text { total }\end{array}$ & $\begin{array}{c}\log _{-} \text {ReceitaMunicipal } \\
\text { stn_hab }\end{array}$ & & & & & & $\begin{array}{l}0,09742 \\
(0.028)^{\star \star *}\end{array}$ \\
\hline Arrecadacão & log_impostos_hab & 0,09448 & 0,07998 & 0,05434 & 0,05459 & 0,05318 & \\
\hline - impostos e & & $(0.012)^{\star \star \star}$ & $(0.012)^{\star \star \star}$ & $(0.008)^{\star \star \star}$ & $(0.007)^{\star * *}$ & $(0.008)^{\star \star \star}$ & \\
\hline transfe- & log_arrecad_transf_ & 0,2742 & 0,12646 & 0,11733 & 0,07921 & 0,07797 & \\
\hline & & $(0.035)^{\star \star \star}$ & $(0.038)^{\star \star \star}$ & $(0.036)^{\star \star \star}$ & $(0.033)^{\star \star \star}$ & $(0.035)^{\star \star}$ & \\
\hline & & & 0,04149 & 0,03498 & 0,02839 & 0,02939 & 0,041275 \\
\hline & & & $(0.026)$ & $(0.027)$ & $(0.025)$ & $(0.025)$ & $(0.024)^{\star}$ \\
\hline & Ing DIR Fotodo hah & & $-0,37201$ & $-0,37052$ & $-0,24786$ & $-0,25221$ & $-0,24445$ \\
\hline & log_rID_Estadu_lla & & $(0.173)^{\star *}$ & $(0.113)^{\star * *}$ & $(0.110)^{\star \star \star}$ & $(0.115)^{\star *}$ & $(0.119)^{\star *}$ \\
\hline enda $a$ & 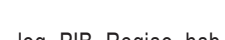 & & 1,80432 & 1,6178 & 1,36713 & 1,37778 & 1,38962 \\
\hline população & log_PIB_Reglao_nab & & $(0.269)^{\star \star *}$ & $(0.160)^{\star \star \star}$ & $(0.168)^{\star \star \star}$ & $(0.174)^{\star \star \star}$ & $(0.177)^{\star \star \star}$ \\
\hline & log $n 0 n$ & & $-0,49668$ & $-0,33383$ & $-0,38365$ & $-0,38653$ & $-0,40515$ \\
\hline & $\log _{\text {log }} p \mathrm{p}$ & & $(0.063)^{\star \star \star}$ & $(0.072)^{\star \star \star}$ & $(0.072)^{\star \star \star}$ & $(0.073)^{\star \star \star}$ & $(0.079)^{\star \star \star}$ \\
\hline & log non vizinhos & & $-0,00718$ & $-0,00245$ & $-0,00048$ & $-0,00009$ & 0,00078 \\
\hline & I0g_pop_víLIIIIOS & & $-0,015$ & $-0,012$ & $-0,012$ & $-0,012$ & $-0,012$ \\
\hline & ner coh nar & & & 0,00353 & 0,00068 & 0,00068 & 0,00065 \\
\hline & PeI_CON_pac & & & $(0.000)^{\star \star \star}$ & $(0.000)^{\star \star *}$ & $(0.005)^{\star \star * *}$ & $(0.0006)^{\star *}$ \\
\hline & nor nah nof & & & 0,00642 & 0,00631 & 0,00627 & 0,00635 \\
\hline Oferta de & per_con_psi & & & $(0.000)^{\star * *}$ & $(0.000)^{\star * *}$ & $(0.000)^{\star \star \star}$ & $(0.000)^{* * *}$ \\
\hline serviços & prod omb ho hot & & & 0,00084 & 0,00077 & 0,00078 & 0,00078 \\
\hline & prou_adin_Das_trado & & & $(0.000)^{\star \star *}$ & $(0.000)^{* * *}$ & $(0.000)^{\star \star *}$ & $(0.003)^{\star \star *}$ \\
\hline & & & & 0,00589 & 0,00636 & 0,00615 & 0,00602 \\
\hline & prod_almo_INDas_man & & & $(0.002)^{\star \star *}$ & $(0.002)^{* * *}$ & $(0.002)^{\star * *}$ & $(0.002)^{* * *}$ \\
\hline & & & & & $-0,00024$ & $-0,00023$ & $-0,00017$ \\
\hline & Miort_5_anos & & & & $(0.001)$ & $(0.001)$ & $(0.001)$ \\
\hline & Pon urh & & & & $-0,00013$ & $-0,00012$ & $-0,00013$ \\
\hline Necessidade & rup_uts & & & & $(0.000)$ & $(0.0003)$ & $(0.0002)$ \\
\hline de saúde & per colato livo & & & & $-0,00033$ & $-0,00038$ & $-0,00032$ \\
\hline & pel_culeta_tixu & & & & $(0.0004)$ & $(0.0005)$ & $(0.0005)$ \\
\hline & & & & & $-0,00072$ & $-0,00074$ & $-0,00076$ \\
\hline & Ix_ODI_Cma & & & & $(0.003)^{\star \star}$ & $(0.0003)^{\star *}$ & $(0.0003)^{\star \star}$ \\
\hline & ind nortidn nroc & & & & & 0,04017 & 0,04067 \\
\hline & Ind_partido_pres & & & & & $(0.015)^{\star * *}$ & $(0.015)^{* \star *}$ \\
\hline Fatores & ind nartidn aov & & & & & 0,00916 & 0,00955 \\
\hline políticos & IIIU_paltiuo_yov & & & & & $-0,028$ & $-0,009$ \\
\hline & ind_partido_aliado & & & & & $-0,00078$ & $-0,00143$ \\
\hline & & & & & & $(0.009)$ & $(0.009)$ \\
\hline & dummies de ano & Sim & Sim & Sim & Sim & Sim & Sim \\
\hline
\end{tabular}

${ }^{*},{ }^{* *},{ }^{* * *}$ : Variável significante a $10 \%, 5 \%$ e $1 \%$ respectivamente.

OBS: Desvios-padrão robustos, entre parêntesis.

Fonte: Dados do estudo. 
Pelo impacto positivo obtido, e de acordo com as regras que determinam as transferências, nossa interpretação é de que quando os municípios arrecadam mais eles também têm maior propensão a investir em programas e serviços que recebem recursos em transferências do SUS. Como comentado anteriormente, nossa hipótese é que os recursos do SUS podem não ser suficientes e, com isso, o investimento em alguns programas e serviços pode exigir dispêndio de recursos próprios, o que tende a ser mais fácil para os municípios quando estes arrecadam mais. Tal argumento ganha força quando se admite que a adesão local a programas do SUS sem o atendimento das metas mínimas exigidas pelo poder central impõe custos ao governo local. É importante notar que uma parte da adesão local a programas específicos do SUS (como o PSF e o PACS) foi controlada na regressão, e, ainda assim, a relação entre arrecadação local e transferêncais fundo a fundo do SUS se mantém, uma vez que não é possível controlar a adesão a todos os programas, como, por exemplo, o programa de combate a carências nutricionais. ${ }^{9}$

Analisando outros aspectos dos resultados, temos que os sinais obtidos para os controles de necessidade de saúde parecem ir na direção contrária. Seria esperado que os municípios com maior taxa de mortalidade infantil e de óbitos por causas mal definidas recebessem maior volume de transferências. Em ambos os casos, os coeficientes encontrados indicam a relação inversa, mas só foram significantes para a taxa de óbitos por causas mal definidas. Esses resultados reforçam a hipótese de Mendes, Miranda e Cossio (Mendes, Miranda e Cossio 2008) de que os critérios de distribuição do SUS parecem não abranger as necessidades de saúde.

Os resultados sugerem ainda que há fatores políticos influenciando a destinação dos recursos do SUS. Segundo as regressões estimadas, nota-se que as transferências SUS para os municípios cujo partido do prefeito é o mesmo que o do presidente foram significativamente maiores.

9 A correlação condicional entre o grau de adesão local ao PSF e ao PACS e o recebimento de transferências SUS per capita é positiva, elevada e estatisticamente significativa, como seria esperado. 


\section{Conclusões}

O Sistema Único de Saúde (SUS) foi concebido a partir da Constituição Federal de 1988, tendo como princípios-chave a universalidade, a equidade e a integralidade na assistência à saúde da população brasileira. Antes do SUS, os recursos federais concentravam-se nas regiões mais desenvolvidas do país e a assistência era restrita à população empregada através da economia formal. Após a implantação do SUS, as transferências intergovernamentais para a saúde têm sido uma ferramenta importante no sentido de promover uma redistribuição de recursos ao longo do território, como também de atribuir maior capacidade e responsabilidade de gestão aos governos locais.

No entanto, este estudo apresenta evidências de que o mecanismo de transferências do SUS pode estar induzindo a uma espécie de "exigência de contrapartida" e a eventuais distorções em seu papel redistributivo, embora não explicitamente. Nesse contexto, o objetivo deste trabalho foi estudar empiricamente os determinantes das transferências intergovernamentais na área da saúde, com foco no aspecto redistributivo, investigando em particular a relação positiva observada entre receita municipal per capita - que inclui a arrecadação local de impostos e o recebimento de transferências incondicionais - e as transferências para a saúde recebidas através da modalidade do SUS conhecida como fundo a fundo. Em princípio essa relação positiva não seria esperada, pois as transferências fundo a fundo do SUS não exigem contrapartidas financeiras.

A análise empírica foi realizada através da estimação de modelos em painel com efeitos fixos e um amplo conjunto de variáveis de controle. Para tanto, utilizou-se dados dos municípios brasileiros no período de 2002 a 2010, obtidos em sua maioria através do Departamento de Informações do SUS (DATASUS) e do Sistema de Informações sobre Orçamentos Públicos em Saúde (SIOPS), ambos do Ministério da Saúde. Como referência teórica, utilizou-se principalmente o modelo apresentado por Chernick (Chernick, 1979), que também trata de transferências condicionais, como é o caso das transferências do SUS. Esse trabalho é citado por Hines e Thaler (Hines e Thaler, 1995) no contexto do flypaper effect, uma vez que a situação estudada caracterizava a existência de correlação positiva entre gastos locais e o recebimento de transferências condicionais. 
Os resultados apontam que a receita municipal per capita exerce, de fato, um impacto positivo e significante sobre o recebimento de transferências fundo a fundo do SUS, tendo sido excluído o montante referente ao Piso de Atenção Básica Fixo, que não depende da adesão a programas específicos. Levantamos a hipótese de que, quando ocorre aumento de receita, os municípios têm mais incentivos para aderir a programas e/ou ofertar serviços que recebem aporte dos recursos do SUS. Argumentamos sobre a possibilidade de que os recursos repassados pelo SUS por unidade de produto não sejam suficientes, de modo que uma contrapartida financeira dos governos locais seria necessária para cobrir os custos dos programas e serviços em sua totalidade. Nesse caso, como os municípios com maior receita têm mais recursos a serem necessariamente destinados à área da saúde, estes teriam mais incentivos a aderir aos programas que recebem transferências SUS, admitindo que a adesão aos programas sem o cumprimento das metas mínimas impõe um custo. Como consequência, seria como se o próprio mecanismo de financiamento do SUS incentivasse os municípios que mais gastam (por terem maior receita municipal) a receberem mais transferências. Outro resultado não esperado é que há fatores políticos influenciando, ao menos em parte, o recebimento de transferências fundo a fundo do SUS, pois os municípios cujos prefeitos são do mesmo partido do presidente recebem em média, segundo as regressões estimadas, um volume maior de recursos.

Pensando apenas no aspecto redistributivo, a relação positiva entre receita municipal e transferências intergovernamentais para a saúde pode não ser desejada. Os municípios com maior receita, em princípio, têm melhores condições de arcar com as necessidades de saúde de sua população. Entretanto, para se ter um parecer mais preciso, seria importante avaliar aspectos relacionados à eficiência da gestão local. Como há evidências de que o SUS não necessariamente premia bons resultados na aplicação de recursos e também não utiliza critérios de necessidade de saúde, o tópico merece atenção e poderia ser explorado em trabalhos futuros, que poderiam investigar também a relação entre receita municipal e as transferências do SUS recebidas através da modalidade de convênios. 


\section{Referências}

BANCO MUNDIAL. Governance in Brazil's Unified Health System (SUS). Relatório do Banco Mundial $n^{\circ}$ 36.601-BR. Washington, 2007.

BRASIL. Constituição. Constituição da República Federativa do Brasil. Brasília, DF: Senado, 1988.

BRASIL. EC-29 é regulamentada. Portal da Saúde, Brasilia - DF, 17 jan. 2012. Disponível em:http:// portalsaude.saude.gov.br/portalsaude/noticia/4001/162/presidenta-dilma-sanciona-lei-que-define-gastos-da-saude.html. Acesso em: 28 out. 2012.

BRASIL. Glossário do Portal Saúde Transparente. Portal Transparência, Brasilia - DF, 2012. Disponível em: http://aplicacao.saude.gov.br/portaltransparencia/common/arquivos/Glossario_Portal_Transparencia.pdf. Acesso em: 28 out. 2012.

BRASIL. Ministério da saúde. Entendendo o SUS. [S.1.], 2006.

BRASIL. Ministério da Saúde. Secretaria de Vigilância à Saúde. Secretaria de Atenção à Saúde. Diretrizes nacionais da vigilância em saúde. In: Série Pactos pela Saúde 2006. Brasília - DF, v. 13, 2010.

BRASIL.Conselho Nacional de Secretários de Saúde. Sistema Único de saúde. In: CONASS (Ed.). Coleção Para Entender a Gestão do SUS. Brasília, v. 1, 2011.

CHERNICK, H. An economic model of the distribution of project grants. In: PETER, M.; WILLIAM, O. (Ed.). Fiscal Federalism and Grants-in-Aid. The Urban Institute, Washington D.C.: [s.n.], p. 81-103, 1979.

COSSIO, F. A. B.; CARVALHO, L. M. Os efeitos expansivos das transferências intergovernamentais e transbordamentos espaciais de despesas públicas: evidências para os municípios brasileiros. In: Pesquisa e Planejamento Econômico. [S.1.: s.n.], v. 31, n. 1, 2001.

CREEMER, J.; ESTACHE, A.; SEABRIGHT, P. Decentralizing public services: what can we learn from the theory of the firm? Revue d'Economie Politique, v. 106, p. 37-60, 1996.

FILIMON, R.; ROMER, T.; ROSENTHAL, H. Asymmetric information and agenda control. Journal of Public Economics, v. 17, p. 51-70, fev. 1982.

HINES, J. R.; THALER, R. H. Anomalies: The flypaper effect. Journal of Economic Perspectives, p. 217-226, 1995.

KNIGHT, B. Endogenous federal grants and crowd-out of state government spending: Theory and evidence from the federal highway aid program. American Economic Review, v. 92, p. 71-92, mar. 2002.

MARCONI, N. et al. Vertical tranfers and the appropriation of resources by the bureaucracy: the case of brazillian state governments. Public Choice, v. 141, p. 65-85, 2009.

MATTOS, E. H. C.; ROCHA, F. F.; ARVATE, P. R. Flypaper effect revisited: Evidence for tax collection efficiency in brazilian municipalities. Estudos Econômicos, v. 41, p. 7-28, 2011.

MENDES, M.; MIRANDA, R. B.; COSSIO, F. Transferências intergovernamentais no Brasil: diagnóstico e proposta de reforma. Consultoria Legislativa do Senado Federal, Texto para Discussão, v. 40 , abr., 2008.

NISKANEN, W. A. Bureaucracy and representative government. Chicago: Aldine-Atherton, 1971.

NUNES, A. A alocação equitativa inter-regional de recursos públicos federais do SUS: a receita própria do município como variável moderadora. Brasília: Ministério da Saúde. (Relatório de Consultoria. Projeto 1.04.21.), 2004.

OATES, W. E. Toward a second-generation theory of fiscal federalism. International Tax and Public Finance, v. 12, p. 349-373, 2005.

PEIXOTO, S.; ROCHA, F.; NISHIJIMA, M.; POSTALI, F. Decentralization and corruption: evidence from primary health-care programmes. Applied Economics Letters, v. 19, p. 1885-1888, 2012. 
PARMAGNANI, F.; ROCHA, F.; Avaliando a resposta dos gastos municipais em saúde às transferências do SUS. 41ํㅡㄹ Encontro Nacional de Economia da ANPEC, 2013.

SAKURAI, S. N. Transferências governamentais e despesas públicas locais: evidências para os municípios brasileiros. In: Informações Fipe. [S.1.: s.n.], 2009. p. - .

SOUZA, R. O Sistema Público de Saúde brasileiro. Seminário Internacional Tendências e Desafios dos Sistemas de Saúde nas Américas, São Paulo, 2002.

VARELA, P. S.; MARTINS, G. de A.; FÁVERO, L. P. L. Production efficiency and financing of public health: an analysis of small municipalities in the state of são paulo brazil. Health Care Management Science, v. 13, p. 112-123, 2010.

WOOLDRIDGE, J. M. Econometric Analysis of cross section and panel data. [S.1.]: MIT Press, 2002.

WYCKOFF, P. G. A bureaucratic theory of flypaper effect. Journal of Urban Economics, v. 23, p. $115-129,1988$.

WYCKOFF, P. G. The elusive flypaper effect. Journal of Urban Economics, v. 30, p. 310-328, 1991. 